\title{
Multi-level Generative Models for Partial Label Learning with Non-random Label Noise
}

\author{
Yan Yan*1, Yuhong Guo ${ }^{2,3}$ \\ ${ }^{1}$ Northwestern Polytechnical University, China \\ ${ }^{2}$ Carleton University, Canada \\ ${ }^{3}$ Canada CIFAR AI Chair, Amii \\ yanyan.nwpu@gmail.com, yuhong.guo@ carleton.ca
}

\begin{abstract}
Partial label (PL) learning tackles the problem where each training instance is associated with a set of candidate labels that include both the true label and some irrelevant noise labels. In this paper, we propose a novel multi-level generative model for partial label learning (MGPLL), which tackles the PL problem by learning both a label level adversarial generator and a feature level adversarial generator under a bi-directional mapping framework between the label vectors and the data samples. MGPLL uses a conditional noise label generation network to model the non-random noise labels and perform label denoising, and uses a multi-class predictor to map the training instances to the denoised label vectors, while a conditional data feature generator is used to form an inverse mapping from the denoised label vectors to data samples. Both the noise label generator and the data feature generator are learned in an adversarial manner to match the observed candidate labels and data features respectively. We conduct extensive experiments on both synthesized and real-world partial label datasets. The proposed approach demonstrates the state-ofthe-art performance for partial label learning.
\end{abstract}

\section{Introduction}

Partial label (PL) learning is a weakly supervised learning problem with ambiguous labels, where each training instance is assigned a set of candidate labels, among which only one is the true label. Since it is typically difficult and costly to annotate instances precisely, the task of partial label learning naturally arises in many real-world learning scenarios, including automatic face naming [Hüllermeier and Beringer, 2006; Zeng et al., 2013], and web mining [Luo and Orabona, 2010].

As the true label information is hidden in the candidate label set, the main challenge of PL lies in identifying the ground truth labels from the candidate noise labels, aiming to learn a good prediction model. Some previous works have made effort on adjusting the existing effective learning techniques to directly handle the candidate label sets and

\footnotetext{
${ }^{*}$ Work conducted while visiting Carleton University.
}

perform label disambiguation implicitly [Gong et al., 2018; Nguyen and Caruana, 2008; Wu and Zhang, 2018]. These methods are good at exploiting the strengths of the standard classification techniques and have produced promising results on PL learning. Another set of works pursue explicit label disambiguation by trying to identify the true labels from the noise labels in the candidate label sets. For example, the work in [Feng and An, 2018] tries to estimate the latent label distribution with iterative label propagations and then induce a prediction model by fitting the learned latent label distribution. Another work in [Lei and An, 2019] exploits a self-training strategy to induce label confidence values and learn classifiers in an alternative manner by minimizing the squared loss between the model predictions and the learned label confidence matrix. However, all these methods have a common drawback: they automatically assumed random noise in the label space - that is, noise labels are randomly distributed in the label space for each instance. However, in real world problems the appearance of noise labels is usually dependent on the target true label. For example, when the object contained in an image is a "computer", a noise label "TV" could possibly be added due to a recognition mistake or image ambiguity, but it is less likely to annotate the object as "lamp" or "curtain", while the probability of getting noise labels such as "tree" or "bike" is even smaller.

In this paper, we propose a novel multi-level adversarial generative model, MGPLL, for partial label learning. The MGPLL model comprises of conditional data generators at both the label level and feature level. The noise label generator directly models non-random appearances of noise labels conditioning on the true label by adversarially matching the candidate label observations, while the data feature generator models the data samples conditioning on the corresponding true labels by adversarially matching the observed data sample distribution. Moreover, a prediction network is incorporated to predict the denoised true label of each instance from its input features, which forms inverse mappings between labels and features, together with the data feature generator. The learning of the overall model corresponds to a minimax adversarial game, which simultaneously identifies true labels of the training instances from both the observed data features and the observed candidate labels, while inducing accurate prediction networks that map input feature vectors to (denoised) true label vectors. To the best of our knowledge, this 
is the first work that exploits multi-level generative models to model non-random noise labels for partial label learning. We conduct extensive experiments on real-world and synthesized PL datasets. The empirical results show the proposed MGPLL achieves the state-of-the-art PL performance.

\section{Related Work}

Partial label (PL) learning is a weakly supervised learning problem, where the true label of each training instance is hidden within a given candidate label set. The challenge of PL learning lies in disambiguating the true labels from the candidate label sets to induce good prediction models.

One strategy towards PL learning is to adjust the standard learning techniques and implicitly disambiguate the noise candidate labels through the statistical prediction pattern of the data. For example, with the maximum likelihood techniques, the likelihood of each PL training sample can be defined over its candidate label set [Liu and Dietterich, 2012]. For the $k$-nearest neighbor technique, the candidate labels from neighbor instances can be aggregated to induce the final prediction on a test instance [Hüllermeier and Beringer, 2006; Gong et al., 2018]. For the maximum margin technique, the classification margin can be defined over the predictive difference between the candidate labels and the non-candidate labels of each training sample [Nguyen and Caruana, 2008; $\mathrm{Yu}$ and Zhang, 2016]. For the boosting technique, the weight of each PL training instance and the confidence value of each candidate label being ground-truth label can be refined via each boosting round [Tang and Zhang, 2017]. For the errorcorrecting output codes technique, multiple binary classifiers are built based on the transformed binary training sets [Zhang et al., 2017]. For the binary decomposition techniques, a onevs-one decomposition strategy has been adopted to address PL learning by considering the relevance of each label pair [Wu and Zhang, 2018].

Recently, there have been increasing attentions in designing explicit feature-aware disambiguation strategies [Feng and An, 2018; Xu et al., 2019a; Feng and An, 2019; Wang et al., 2019a]. The authors of [Feng and An, 2018] attempt to refine the latent label distribution using iterative label propagations and then induce a predictive model based on the learned latent label distribution. Another work in [Lei and An, 2019] tries to refine the label confidence values with a self-training strategy and induce the prediction model over the refined label confidence scores via alternative optimization. A recent work in [Yao et al., 2020] proposes to address the PL learning problem by enhancing the representation ability via deep features and improving the discrimination ability through margin maximization between the candidate labels and the non-candidate labels. Another recent work in [Yan and Guo, 2020] proposes to dynamically correct label confidence values with a batch-wise label correction strategy and induce a robust predictive model based on the MixUp enhanced data. Although these works demonstrate good empirical performance, they are subject to one common drawback of assuming random distributions of noise labels by default, which may not hold in many real-world learning scenarios. This paper presents the first work that explicitly model non- random noise labels for partial label learning.

PL learning is related to other types of weakly supervised learning problems, including noise label learning (NLL) [Xu et al., 2019b; Thekumparampil et al., 2018] and partial multilabel learning (PML) [Wang et al., 2019b; Xie and Huang, 2018; Yan and Guo, 2021], but addresses different problems from them. The main difference between the PL learning and the other two well-established learning problems lies in the assumption on the label information provided by the training samples. Both PL learning and NLL aim to induce a multiclass prediction model from the training instances with noisecorrupted labels. However NLL assumes the true labels on some training instances are replaced by the noise labels, while PL assumes the true-label coexists with the noise labels in the candidate label set of each training instance. Hence the off-the-shelf NLL learning methods cannot be directly applied to solve the PL learning problem. Both PL learning and PML learn from training samples with ambiguous candidate label sets, which contain the true labels and additional noise labels. But PL learning addresses a multi-class learning problem where each candidate label set contains only one true label, while PML learning addresses a multi-label learning problem where each candidate label set contains all the true labels whose number is unknown.

The Wasserstein Generative Adversarial Network (WGAN) [Arjovsky et al., 2017] is a popular alternative to the standard GAN [Goodfellow et al., 2014] due to its effectiveness and stableness in training. During the past few years, WGANs have been proposed as a successful tool for various applications, including adversarial sample generation [Zhao et al., 2017], domain adaption [Dou et al., 2018], and learning with noisy labels [Chen et al., 2018]. This paper presents the first work that exploits WGAN to model non-random noise labels for partial label learning.

\section{Proposed Approach}

Given a partial label training set $S=\left\{\left(\mathbf{x}_{i}, \mathbf{y}_{i}\right)\right\}_{i=1}^{N}$, where $\mathbf{x}_{i} \in \mathbb{R}^{d}$ is a $d$-dimensional feature vector for the $i$-th instance, and $\mathbf{y}_{i} \in\{0,1\}^{L}$ denotes the candidate label indicator vector associated with $\mathbf{x}_{i}$, which has multiple 1 values corresponding to the ground-truth label and the additional noise labels, the task of PL learning is to learn a good multiclass prediction model from $S$. In real world scenarios, the irrelevant noise labels are typically not presented in a random manner, but rather correlated with the ground-truth label. In this section, we present a novel multi-level generative model for partial label learning, MGPLL, which models nonrandom noise labels using an adversarial conditional noise label generator, and builds connections between the denoised label vectors and instance features using a label-conditioned feature generator and a label prediction network. The overall model learning problem corresponds to a minimax adversarial game, which conducts multi-level generator learning by matching the observed data in both the feature and label spaces, while boosting the correspondence relationships between features and labels to induce an accurate multi-class prediction model.

Figure 1 illustrates the proposed multi-level generative 


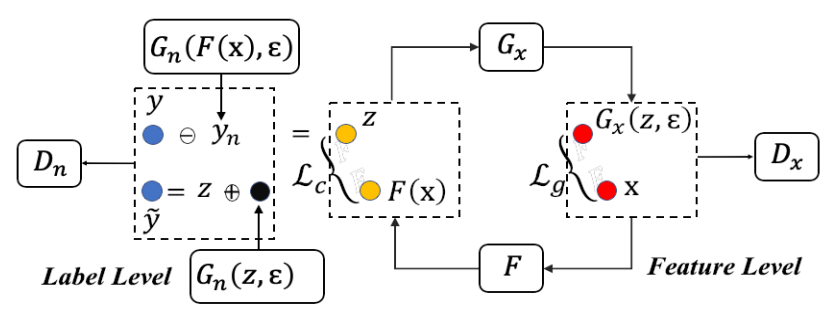

Figure 1: The proposed MGPLL model. It consists of an adversarial generative model at the label level with a conditional noise label generator $G_{n}$ and a discriminator $D_{n}$, and an adversarial generative model at the feature level with a conditional sample generator $G_{x}$ and a discriminator $D_{x}$. The prediction network $F$ builds connections between these two level generative models while providing an inverse mapping for $G_{x}$.

model, MGPLL, which attempts to address the partial label learning problem from both the label level and feature level under a bi-directional mapping framework. The MGPLL model comprises five component networks, which are simple multilayer perceptrons: the conditional noise label generator, $G_{n}$, which models the noise labels conditioning on the ground-truth label at the label level; the conditional data generator, $G_{x}$, which generates data samples at the feature level conditioning on the denoised label vectors; the discriminator, $D_{n}$, which separates the generated candidate label vectors from the observed candidate label vectors in the real training data; the discriminator, $D_{x}$, which separates the generated samples from the real data in the feature space; and the prediction network, $F$, which predicts the denoised label for each sample from its input features. The conditional noise label generator $G_{n}$ induces the denoised prediction target for the prediction network $F$, while the conditional data generator $G_{x}$ learns an inverse mapping at the feature level that maps the denoised label vectors in the label space to the data samples in the feature space. Below we present the details of the two level generations and the overall learning algorithm.

\subsection{Conditional Noise Label Generation}

The key challenge of partial label learning lies in the fact that the ground-truth label is hidden among the noise labels in the given candidate label set. As aforementioned, in real world partial label learning problems, the presence of noise labels typically does not happen at random, but rather correlates with the ground-truth labels. Hence we propose a conditional noise label generation model to model the appearances of the target-label dependent noise labels by adversarially matching the observed candidate label distribution in the training data, aiming to help identify the true labels later.

Specifically, given a noise value sampled from a uniform distribution $\epsilon \sim P_{\epsilon}$ and a one-hot label indicator vector $\mathbf{z}$ sampled from a multinomial distribution $P_{\mathbf{z}}$, we use a noise label generator $G_{n}(\mathbf{z}, \epsilon)$ to generate a noise label vector conditioning on the true label $\mathbf{z}$, which can be combined with $\mathbf{z}$ in a rectified sum, " $\oplus$ ", to form a generated candidate label vector $\tilde{\mathbf{y}}$, such that

$$
\tilde{\mathbf{y}}=G_{n}(\mathbf{z}, \epsilon) \oplus \mathbf{z}=\min \left(G_{n}(\mathbf{z}, \epsilon)+\mathbf{z}, 1\right) .
$$

Here we assume the generator $G_{n}$ generates non-negative values. We then adopt the adversarial learning principle to learn such a noise label generation model by introducing a discriminator $D_{n}(\mathbf{y})$, which is a two-class classifier and predicts how likely a given label vector $\mathbf{y}$ comes from the real data instead of the generated data. By adopting the adversarial loss of the Wasserstein Generative Adversarial Network (WGAN), our adversarial learning problem can be formulated as the following minimax optimization problem:

$$
\begin{aligned}
\min _{G_{n}} \max _{D_{n}} & \mathcal{L}_{a d v}^{n}\left(G_{n}, D_{n}\right)= \\
& \mathbb{E}_{\left(\mathbf{x}_{i}, \mathbf{y}_{i}\right) \sim S} D_{n}\left(\mathbf{y}_{i}\right)-\underset{\substack{\mathbf{z} \sim P_{\mathbf{z}} \\
\epsilon \sim P_{\epsilon}}}{\mathbb{E}_{\mathbf{m}}} D_{n}\left(G_{n}(\mathbf{z}, \epsilon) \oplus \mathbf{z}\right)
\end{aligned}
$$

Here the discriminator $D_{n}$ attempts to maximally distinguish the generated candidate label vectors from the observed candidate label indicator vectors in the real training data, while the generator $G_{n}$ tries to generate noise label vectors and hence candidate label vectors that are similar to the real data in order to maximally confuse the discriminator $D_{n}$. By playing a minimax game between the generator $G_{n}$ and the discriminator $D_{n}$, the adversarial learning is expected to induce a generator $G_{n}^{*}$ such that the generated candidate label distribution can match the observed candidate label distribution in the training data. We adopt the training loss of the WGAN here, as WGANs can overcome the mode collapse problem and have improved learning stability comparing to the standard GAN models [Arjovsky et al., 2017].

Note although the proposed generator $G_{n}$ is designed to model true-label dependent noise labels, it can be easily modified to model random noise label distributions by simply dropping the label vector input, which yields $G_{n}(\epsilon)$.

\subsection{Prediction Network}

The ultimate goal of partial label learning is to learn an accurate prediction network $F$. To train a good predictor, we need to obtain denoised labels on the training data. For a candidate label indicator vector $\mathbf{y}$, if the noise label indicator vector $\mathbf{y}_{n}$ is given, one can simply perform label denoising as follows to obtain the corresponding true label vector $\mathbf{z}$ :

$$
\mathbf{z}=\mathbf{y} \ominus \mathbf{y}_{n}=\max \left(\mathbf{y}-\mathbf{y}_{\mathbf{n}}, 0\right)
$$

Here the rectified minus operator " $\ominus$ " is introduced to generalize the standard minus "-_" operator into the non-ideal case, where the noise label indicator vector $\mathbf{y}_{n}$ is not properly contained in the candidate label indicator vector.

The generator $G_{n}$ presented in the previous section provides a mechanism to generate noise labels and denoise candidate label sets, but requires the true target label vector as its input. We propose to use the outputs of the prediction network $F$ to approximate the target true label vectors of the training data for the purpose of denoising the candidate labels with $G_{n}$, while using the denoised labels as the prediction target for $F$. Specifically, with the noise label generator $G_{n}$ and the predictor $F$, we perform partial label learning by minimizing the following classification loss on the training data:

$\min _{F, G_{n}} \mathcal{L}_{c}\left(F, G_{n}\right)=\mathbb{E}_{\substack{\epsilon \sim P_{\epsilon} \\\left(\mathbf{x}_{i}, \mathbf{y}_{i}\right) \sim S}} \ell_{c}\left(F\left(\mathbf{x}_{i}\right), \mathbf{y}_{i} \ominus G_{n}\left(F\left(\mathbf{x}_{i}\right), \epsilon\right)\right)$ 
Although in the ideal case, the output vectors of $G_{n}$ and $F$ would be indicator label vectors, it is error-prone and difficult for neural networks to output discrete values. To pursue more reliable predictions and avoid overconfident outputs, we use $G_{n}$ and $F$ to predict the probability of each class label being a noise label and the ground-truth label respectively. Hence the loss function $\ell_{c}(\cdot, \cdot)$ in Eq.(4) above denotes a mean square error loss between the predicted probability of each label being the true label (through $F$ ) and its denoised confidence of being a ground-truth label (through $G_{n}$ ).

\subsection{Conditional Feature Level Data Generation}

With the noise label generation model and the prediction network above, the observed training data in both the label and feature spaces are exploited to recognize the true labels and induce good prediction models. Next, we incorporate a conditional data generator $G_{x}(\mathbf{z}, \epsilon)$ at the feature level to map (denoised) label vectors in the label space into instances in the feature space, aiming to further strengthen the mapping relations between data samples and the corresponding labels, enhance label denoising and hence improve the partial label learning performance. Specifically, given a noise value $\epsilon$ sampled from a uniform distribution $P_{\epsilon}$ and a one-hot label vector $\mathbf{z}$ sampled from a multinomial distribution $P_{\mathbf{z}}, G_{x}(\mathbf{z}, \epsilon)$ generates an instance in the feature space that is corresponding to label $\mathbf{z}$. Given the training label vectors in $S$ denoised with $G_{n}$, the data generator $G_{x}$ is also expected to regenerate the corresponding training instances in the feature space. This assumption can be captured using a generation loss:

$$
\begin{array}{r}
\mathcal{L}_{g}\left(F, G_{n}, G_{x}\right)=\mathbb{E}_{\substack{\left(\mathbf{x}_{i}, \mathbf{y}_{i}\right) \sim S \\
\epsilon_{1}, \epsilon_{2} \sim P_{\epsilon}}} \ell_{g}\left(G_{x}\left(\mathbf{z}_{i}, \epsilon_{2}\right), \mathbf{x}_{i}\right) \\
\text { with } \quad \mathbf{z}_{i}=\mathbf{y}_{i} \ominus G_{n}\left(F\left(\mathbf{x}_{i}\right), \epsilon_{1}\right)
\end{array}
$$

where $\mathbf{z}_{i}$ denotes the denoised label vector for the $i$-th training instance, and $\ell_{g}(\cdot, \cdot)$ is a mean square error loss function.

Moreover, by introducing a discriminator $D_{x}(\mathbf{x})$, which predicts how likely a given instance $\mathbf{x}$ is real, we can deploy an adversarial learning scheme to learn the generator $G_{x}$ through the following minimax optimization problem with the WGAN loss:

$$
\begin{aligned}
\min _{G_{x}} \max _{D_{x}} & \mathcal{L}_{a d v}^{x}\left(G_{x}, D_{x}\right)= \\
& \mathbb{E}_{\left(\mathbf{x}_{i}, \mathbf{y}_{i}\right) \sim S} D_{x}\left(\mathbf{x}_{i}\right)-\underset{\substack{\mathbf{z} \sim P_{\mathbf{z}} \\
\epsilon \sim P_{\epsilon}}}{\mathbb{E}} D_{x}\left(G_{x}(\mathbf{z}, \epsilon)\right)
\end{aligned}
$$

By playing a minimax game between $G_{x}$ and $D_{x}$, this adversarial learning is expected to induce a generator $G_{x}^{*}$ that can generate samples with the same distribution as the observed training instances. Together with the generation loss in Eq.(5), we expect the mapping relation from label vectors to samples induced by $G_{x}^{*}$ can be consistent with the observed data. Moreover, the consistency of the mapping relation induced by $G_{x}$ and the inverse mapping from samples to label vectors through the prediction network $F$ can be further strengthened by enforcing an auxiliary classification loss on the generated data:

$$
\mathcal{L}_{c^{\prime}}\left(F, G_{x}\right)=\mathbb{E}_{\substack{\mathbf{z} \sim P_{\mathbf{z}} \\ \epsilon \sim P_{\epsilon}}} \ell_{c^{\prime}}\left(F\left(G_{x}(\mathbf{z}, \epsilon)\right), \mathbf{z}\right)
$$

where $\ell_{c^{\prime}}(\cdot, \cdot)$ can be a cross-entropy loss between the label prediction probability vector and the sampled true label indicator vector.

\subsection{Learning the MGPLL Model}

By integrating the classification loss in Eq.(4), the adversarial losses in Eq.(2) and Eq.(6), the generation loss in Eq.(5) and the auxiliary classification loss in Eq.(7) together, MGPLL learning can be formulated as the following min-max optimization problem:

$$
\begin{aligned}
\min _{G_{n}, G_{x}, F} \max _{D_{n}, D_{x}} \mathcal{L}_{c}\left(F, G_{n}\right)+\mathcal{L}_{a d v}^{n}\left(G_{n}, D_{n}\right)+ \\
\alpha \mathcal{L}_{a d v}^{x}\left(G_{x}, D_{x}\right)+\beta \mathcal{L}_{g}\left(F, G_{n}, G_{x}\right)+\gamma \mathcal{L}_{c^{\prime}}\left(F, G_{x}\right)
\end{aligned}
$$

where $\alpha, \beta$ and $\gamma$ are trade-off hyperparameters. The learning of the overall model corresponds to a minimax adversarial game. We develop a batch-based stochastic gradient descent algorithm to solve it by conducting minimization over $\left\{G_{n}, G_{x}, F\right\}$ and maximization over $\left\{D_{n}, D_{x}\right\}$ alternatively.

\section{Experiment}

We conducted extensive experiments on both controlled synthetic PL datasets and real-world PL datasets to investigate the empirical performance of the proposed model. In this section, we present our experimental settings, comparison results and discussions.

\subsection{Experiment Setting \\ Datasets}

The synthetic datasets are generated from six UCI datasets, ecoli, deter, vehicle, segment, satimage and letter. From each UCI dataset, we generated synthetic PL datasets using three controlling parameters $p, r$ and $\epsilon$, following the controlling protocol in previous studies [Wu and Zhang, 2018; Lei and An, 2019]. Among the three parameters, $p$ controls the proportion of instances that have noise candidate labels, $r$ controls the number of false positive labels, and $\epsilon$ controls the probability of a specific false positive label co-occurring with the true label. Under different parameter configurations, multiple PL variants can be generated from each UCI dataset. Given that both random noise labels and target label-dependent noise labels may exist in real-world applications, we considered two types of settings. In the first type of setting, we consider random noise labels with the following three groups of configurations: (I) $r=1$, $p \in\{0.1,0.2, \cdots, 0.7\}$; (II) $r=2, p \in\{0.1,0.2, \cdots, 0.7\}$; and (III) $r=3, p \in\{0.1,0.2, \cdots, 0.7\}$. In the second type of setting, we consider the target label-dependent noise labels with the following configuration: (IV) $p=1, r=1$, $\epsilon \in\{0.1,0.2, \cdots, 0.7\}$. In total, the four groups of configurations provide us 168 ( 28 configurations $\times 6$ UCI datasets) synthetic PL datasets.

We used five real-world PL datasets that are collected from several application domains, including FG-NET [Panis and Lanitis, 2014] for facial age estimation, Lost [Cour et al., 2011], Yahoo! News [Guillaumin et al., 2010] for automatic face naming in images or videos, MSRCv2 [Dietterich and Bakiri, 1994] for object classification, and BirdSong [Briggs et al., 2012] for bird song classification.

\section{Comparison Methods}

We compared the proposed MGPLL with the following PL methods, each configured with parameters according to the 


\begin{tabular}{l|ccccc}
\hline & \multicolumn{5}{|c}{ MGPLL vs - } \\
& SURE & PALOC & CLPL & PL-SVM & PL-KNN \\
\hline varying $p[r=1]$ & $25 / 14 / 3$ & $32 / 10 / 0$ & $36 / 6 / 0$ & $37 / 5 / 0$ & $37 / 5 / 0$ \\
varying $p[r=2]$ & $27 / 13 / 2$ & $33 / 9 / 0$ & $33 / 9 / 0$ & $38 / 4 / 0$ & $35 / 7 / 0$ \\
varying $p[r=3]$ & $26 / 14 / 2$ & $32 / 10 / 0$ & $32 / 10 / 0$ & $36 / 6 / 0$ & $34 / 8 / 0$ \\
varying $\epsilon[p, r=1]$ & $25 / 17 / 0$ & $30 / 12 / 0$ & $32 / 10 / 0$ & $35 / 7 / 0$ & $33 / 9 / 0$ \\
\hline Total & $\mathbf{1 0 3 / 5 8 / 7}$ & $\mathbf{1 2 7 / 4 1 / 0}$ & $\mathbf{1 3 3 / 3 5 / 0}$ & $\mathbf{1 4 6 / 2 2 / 0}$ & $\mathbf{1 3 9 / 2 9 / 0}$ \\
\hline
\end{tabular}

Table 1: Win/tie/loss counts of pairwise t-test (at 0.05 significance level) between MGPLL and each comparison method.

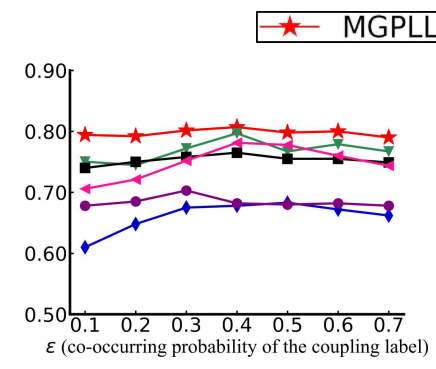

(a) vehicle

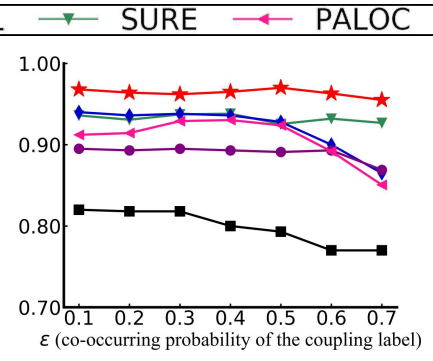

(b) segment

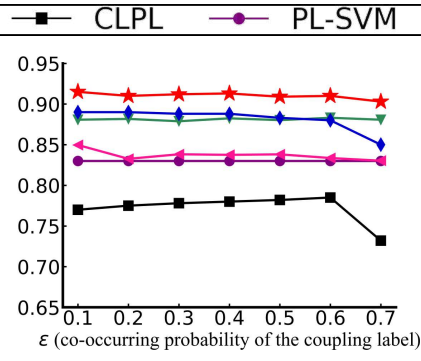

(c) satimage

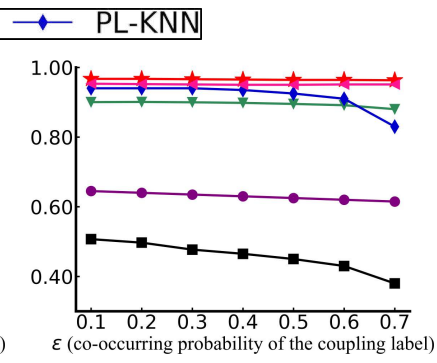

(d) letter

Figure 2: Test accuracy of each comparison method as $\epsilon$ increases from 0.1 to 0.7 (with $100 \%$ partially labeled examples $[p=1]$ and one false positive candidate label $[r=1]$ ).

respective literature: PL-KNN [Hüllermeier and Beringer, 2006], PL-SVM [Nguyen and Caruana, 2008], CLPL [Cour et al., 2011], PALOC [Wu and Zhang, 2018], and SURE [Lei and An, 2019].

\subsection{Results on Synthetic PL Datasets}

We conducted experiments on two types of synthetic PL datasets generated from the UCI datasets, with random noise labels and target label-dependent noise labels, respectively. For each PL dataset, ten-fold cross-validation is performed and the average test accuracy results are recorded. Figure 2 presents the comparison results for the configuration setting (IV) on four datasets. We can see that the proposed MGPLL consistently outperforms all the other methods.

To statistically study the significance of the performance gains achieved by MGPLL over the other comparison methods, we conducted pairwise t-test at 0.05 significance level based on the comparison results of ten-fold cross-validation over all the 168 synthetic PL datasets obtained from all the different configuration settings. The detailed win/tie/loss counts between MGPLL and each comparison method are reported in Table 1. From the results, we have the following observations: (1) MGPLL achieves superior or at least comparable performance against PALOC, CLPL, PL-SVM and PL-KNN in all cases, which is not easy given the comparison methods have different strengths across different datasets. (2) MGPLL significantly outperforms PALOC, CLPL, PLSVM and PL-KNN in $75.6 \%, 79.1 \%, 86.9 \%$ and $82.7 \%$ of the cases respectively, and produces ties in the remaining cases. (3) MGPLL significantly outperforms SURE in $61.3 \%$ of the cases, achieves comparable performance with SURE in $34.5 \%$ of the cases, while being outperformed by SURE in only $4.2 \%$ of the cases. (4) On the PL datasets with target label-dependent noise labels, we can see that MGPLL significantly outperforms SURE, PALOC, CLPL, PL-SVM, PL$\mathrm{KNN}$ in $59.5 \%, 71.4 \%, 76.2 \%, 83.3 \%, 78.6 \%$ of the cases respectively. (5) It is worth noting that MGPLL is never significantly outperformed by any comparison method on datasets with label-dependent noise labels. In summary, these results on the controlled PL datasets clearly demonstrate the effectiveness of MGPLL for PL learning under different settings.

\subsection{Results on Real-World PL Datasets}

We compared the proposed MGPLL method with the comparison methods on five real-world PL datasets. For each dataset, ten-fold cross-validation is conducted. The mean test accuracy and the standard deviation results are reported in Table 2. Moreover, statistical pairwise t-test at 0.05 significance level is conducted to compare MGPLL with each comparison method based on the results of ten-fold cross-validation. The significance results are indicated in Table 2 as well. Note that the average number of candidate labels (avg.\#CLs) of FGNET dataset is quite large, which causes poor performance for all the comparison methods. For better evaluation of this facial age estimation task, we employ the conventional mean absolute error (MAE) [Zhang et al., 2016] to conduct two extra experiments. Two extra test accuracies are reported on the FG-NET dataset where a test sample is considered to be correctly predicted if the difference between the predicted age and the ground-truth age is less than 3 years (MAE3) or 5 years (MAE5). From Table 2 we have the following observations: (1) Comparing with all the other five PL methods, MGPLL consistently produces the best results on all the datasets, with remarkable performance gains in many cases. For example, MGPLL outperforms the best alternative comparison methods by $5.2 \%, 3.4 \%$ and $2.0 \%$ on MSRCv2, Yahoo! News 


\begin{tabular}{l|l|l|l|l|l|l}
\hline & MGPLL & SURE & PALOC & CLPL & PL-SVM & PL-KNN \\
\hline FG-NET & $0.079_{ \pm 0.024}$ & $0.068_{ \pm 0.032}$ & $0.064_{ \pm 0.019}$ & $0.063_{ \pm 0.027}$ & $0.063_{ \pm 0.029}$ & $0.038_{ \pm 0.025} \bullet$ \\
FG-NET(MAE3) & $0.468_{ \pm 0.027}$ & $0.458_{ \pm 0.024}$ & $0.435_{ \pm 0.018} \bullet$ & $0.458_{ \pm 0.022}$ & $0.356_{ \pm 0.022} \bullet$ & $0.269_{ \pm 0.045} \bullet$ \\
FG-NET(MAE5) & $0.626_{ \pm 0.022}$ & $0.615_{ \pm 0.019}$ & $0.609_{ \pm 0.043} \bullet$ & $0.596_{ \pm 0.017} \bullet$ & $0.479_{ \pm 0.016} \bullet$ & $0.438_{ \pm 0.053} \bullet$ \\
Lost & $0.798_{ \pm 0.033}$ & $0.780_{ \pm 0.036}$ & $0.629_{ \pm 0.056} \bullet$ & $0.742_{ \pm 0.038} \bullet$ & $0.729_{ \pm 0.042} \bullet$ & $0.424_{ \pm 0.036} \bullet$ \\
MSRCv2 & $0.533_{ \pm 0.021}$ & $0.481_{ \pm 0.036} \bullet$ & $0.479_{ \pm 0.042} \bullet$ & $0.413_{ \pm 0.041} \bullet$ & $0.461_{ \pm 0.046} \bullet$ & $0.448_{ \pm 0.037} \bullet$ \\
BirdSong & $0.748_{ \pm 0.020}$ & $0.728_{ \pm 0.024} \bullet$ & $0.711_{ \pm 0.016} \bullet$ & $0.632_{ \pm 0.019} \bullet$ & $0.660_{ \pm 0.037} \bullet$ & $0.614_{ \pm 0.021} \bullet$ \\
Yahoo! News & $0.678_{ \pm 0.008}$ & $0.644_{ \pm 0.015} \bullet$ & $0.625_{ \pm 0.005} \bullet$ & $0.462_{ \pm 0.009} \bullet$ & $0.629_{ \pm 0.010} \bullet$ & $0.457_{ \pm 0.004} \bullet$ \\
\hline
\end{tabular}

Table 2: Test accuracy (mean \pm std) of each comparison method on the real-world PL datasets. •/० indicates whether MGPLL is statistically superior/inferior to the comparison method on each dataset (pairwise t-test at 0.05 significance level).

\begin{tabular}{l|l|l|l|l|l|l}
\hline & MGPLL & CLS-w/o-advn & CLS-w/o-advx & CLS-w/o-g & CLS-w/o-aux & CLS \\
\hline FG-NET & $0.079_{ \pm 0.024}$ & $0.061_{ \pm 0.024}$ & $0.072_{ \pm 0.020}$ & $0.068_{ \pm 0.029}$ & $0.076_{ \pm 0.022}$ & $0.057_{ \pm 0.016}$ \\
FG-NET(MAE3) & $0.468_{ \pm 0.027}$ & $0.430_{ \pm 0.029}$ & $0.451_{ \pm 0.032}$ & $0.436_{ \pm 0.038}$ & $0.456_{ \pm 0.033}$ & $0.420_{ \pm 0.420}$ \\
FG-NET(MAE5) & $0.626_{ \pm 0.022}$ & $0.583_{ \pm 0.055}$ & $0.605_{ \pm 0.031}$ & $0.590_{ \pm 0.045}$ & $0.612_{ \pm 0.044}$ & $0.570_{ \pm 0.034}$ \\
Lost & $0.798_{ \pm 0.033}$ & $0.623_{ \pm 0.037}$ & $0.754_{ \pm 0.032}$ & $0.687_{ \pm 0.026}$ & $0.782_{ \pm 0.043}$ & $0.609_{ \pm 0.040}$ \\
MSRCv2 & $0.533_{ \pm 0.021}$ & $0.472_{ \pm 0.030}$ & $0.480_{ \pm 0.038}$ & $0.497_{ \pm 0.031}$ & $0.526_{ \pm 0.036}$ & $0.450_{ \pm 0.037}$ \\
BirdSong & $0.748_{ \pm 0.020}$ & $0.728_{ \pm 0.010}$ & $0.732_{ \pm 0.011}$ & $0.716_{ \pm 0.011}$ & $0.742_{ \pm 0.024}$ & $0.6744_{ \pm 0.016}$ \\
Yahoo! News & $0.678_{ \pm 0.008}$ & $0.645_{ \pm 0.008}$ & $0.675_{ \pm 0.009}$ & $0.648_{ \pm 0.014}$ & $0.671_{ \pm 0.012}$ & $0.610_{ \pm 0.015}$ \\
\hline
\end{tabular}

Table 3: Comparison results of MGPLL and its five ablation variants.

and Birdsong respectively. (2) Out of the total 35 comparison cases ( 5 comparison methods $\times 7$ datasets), MGPLL significantly outperforms all the comparison methods across $77.1 \%$ of the cases, and achieves competitive performance in the remaining $22.9 \%$ of cases. (3) It is worth noting that the performance of MGPLL is never significantly inferior to any other comparison method. These results again validate the efficacy of the proposed method.

\subsection{Ablation Study}

The objective function of MGPLL contains five loss terms: classification loss, adversarial loss at the label level, adversarial loss at the feature level, generation loss and auxiliary classification loss. To assess the contribution of each part, we conducted an ablation study by comparing MGPLL with the following ablation variants: (1) CLS-w/o-advn, which drops the adversarial loss at the label level. (2) CLS-w/oadvx, which drops the adversarial loss at the feature level. (3) CLS-w/o-g, which drops the generation loss. (4) CLS-w/oaux, which drops the auxiliary classification loss. (5) CLS, which only uses the classification loss by dropping all the other loss terms. The comparison results are reported in Table 3. We can see that comparing to the full model, all five variants produce inferior results in general and have performance degradations to different degrees. This demonstrates that the different components in MGPLL all contribute to the proposed model to some extend. From Table 3, we can also see that the variant CLS-w/o-advn has a relatively larger performance degradation by dropping the adversarial loss at the label level, while the variant CLS-w/o-aux has a small performance degradation by dropping the auxiliary classification loss. This makes sense as by dropping the adversarial loss for learning noise label generator, the generator can produce poor predictions and seriously impact the label denoising of the
MGPLL model. This suggests that our non-random noise label generation through adversarial learning is a very effective and important component for MGPLL. For CLS-w/o-aux, as we have already got the classification loss on real data, it is reasonable to see that the auxiliary classification loss on generated data can help but is not critical. Overall, the ablation results suggest that the proposed MGPLL is effective.

\section{Conclusion}

In this paper, we proposed a novel multi-level generative model, MGPLL, for partial label learning. MGPLL uses a conditional label level generator to model the target label dependent non-random noise label appearances and perform candidate label denoising, while using a conditional feature level generator to generate data samples from denoised label vectors. Moreover, a prediction network is incorporated to predict the denoised true label of each instance from its input features, which forms bi-directional inverse mappings between labels and features, together with the data feature generator. The adversarial learning of the overall model simultaneously identifies true labels of the training instances from both the observed data features and the observed candidate labels, while inducing an accurate prediction network. We conducted extensive experiments on real-world and synthesized PL datasets. The proposed MGPLL model demonstrates the state-of-the-art PL performance.

\section{Acknowledgements}

This research was supported in part by the NSERC Discovery Grant, the Canada Research Chairs Program, the Canada CIFAR AI Chairs Program, and the China Scholarship Council. 


\section{References}

[Arjovsky et al., 2017] Martin Arjovsky, Soumith Chintala, and Léon Bottou. Wasserstein generative adversarial networks. In Proc. of ICML, 2017.

[Briggs et al., 2012] Forrest Briggs, Xiaoli Z Fern, and Raviv Raich. Rank-loss support instance machines for MIML instance annotation. In Proc. of KDD, 2012.

[Chen et al., 2018] Jingwen Chen, Jiawei Chen, Hongyang Chao, and Ming Yang. Image blind denoising with generative adversarial network based noise modeling. In Proc. of CVPR, 2018.

[Cour et al., 2011] Timothee Cour, Ben Sapp, and Ben Taskar. Learning from partial labels. Journal of Machine Learning Research, 12(May):1501-1536, 2011.

[Dietterich and Bakiri, 1994] Thomas G Dietterich and Ghulum Bakiri. Solving multiclass learning problems via error-correcting output codes. JAIR, 2:263-286, 1994.

[Dou et al., 2018] Q. Dou, C. Ouyang, Ch. Chen, H. Chen, and P. Heng. Unsupervised cross-modality domain adaptation of convnets for biomedical image segmentations with adversarial loss. arXiv preprint arXiv:1804.10916, 2018.

[Feng and An, 2018] Lei Feng and Bo An. Leveraging latent label distributions for partial label learning. In Proc. of IJCAI, 2018.

[Feng and An, 2019] Lei Feng and Bo An. Partial label learning by semantic difference maximization. In Proc. of IJCAI, 2019.

[Gong et al., 2018] Chen Gong, Tongliang Liu, Yuanyan Tang, Jian Yang, Jie Yang, and Dacheng Tao. A regularization approach for instance-based superset label learning. IEEE transactions on cybernetics, 48(3):967-978, 2018.

[Goodfellow et al., 2014] Ian Goodfellow, Jean PougetAbadie, Mehdi Mirza, Bing Xu, David Warde-Farley, Sherjil Ozair, Aaron Courville, and Yoshua Bengio. Generative adversarial nets. In Proc. of NeurIPS, 2014.

[Guillaumin et al., 2010] Matthieu Guillaumin, Jakob Verbeek, and Cordelia Schmid. Multiple instance metric learning from automatically labeled bags of faces. In Proc. of ECCV, 2010.

[Hüllermeier and Beringer, 2006] E. Hüllermeier and J. Beringer. Learning from ambiguously labeled examples. Intelligent Data Analy., 10(5):419-439, 2006.

[Lei and An, 2019] Feng Lei and Bo An. Partial label learning with self-guided retraining. In Proc. of AAAI, 2019.

[Liu and Dietterich, 2012] Liping Liu and Thomas G Dietterich. A conditional multinomial mixture model for superset label learning. In Proc. of NeurIPS, 2012.

[Luo and Orabona, 2010] Jie Luo and Francesco Orabona. Learning from candidate labeling sets. In Proc. of NeurIPS, 2010.

[Nguyen and Caruana, 2008] Nam Nguyen and Rich Caruana. Classification with partial labels. In $K D D, 2008$.
[Panis and Lanitis, 2014] Gabriel Panis and Andreas Lanitis. An overview of research activities in facial age estimation using the FG-NET aging database. In Proc. of ECCV, 2014.

[Tang and Zhang, 2017] Cai-Zhi Tang and Min-Ling Zhang. Confidence-rated discriminative partial label learning. In Proc. of AAAI, 2017.

[Thekumparampil et al., 2018] Kiran K Thekumparampil, Ashish Khetan, Zinan Lin, and Sewoong Oh. Robustness of conditional gans to noisy labels. In NeurIPS, 2018.

[Wang et al., 2019a] Deng-Bao Wang, Li Li, and Min-Ling Zhang. Adaptive graph guided disambiguation for partial label learning. In Proc. of KDD, 2019.

[Wang et al., 2019b] Haobo Wang, Weiwei Liu, Yang Zhao, Chen Zhang, Tianlei Hu, and Gang Chen. Discriminative and correlative partial multi-label learning. In Proc. of IJCAI, 2019.

[Wu and Zhang, 2018] Xuan Wu and Min-Ling Zhang. Towards enabling binary decomposition for partial label learning. In Proc. of IJCAI, 2018.

[Xie and Huang, 2018] Ming-Kun Xie and Sheng-Jun Huang. Partial multi-label learning. In AAAI, 2018.

[Xu et al., 2019a] Ning Xu, Jiaqi Lv, and Xin Geng. Partial label learning via label enhancement. In $A A A I, 2019$.

[Xu et al., 2019b] Yilun Xu, Peng Cao, Yuqing Kong, and Yizhou Wang. L_DMI: A novel information-theoretic loss function for training deep nets robust to label noise. In Proc. of NeurIPS, 2019.

[Yan and Guo, 2020] Yan Yan and Yuhong Guo. Partial label learning with batch label correction. In $A A A I, 2020$.

[Yan and Guo, 2021] Yan Yan and Yuhong Guo. Adversarial partial multi-label learning with label disambiguation. In AAAI, 2021.

[Yao et al., 2020] Yao Yao, Chen Gong, Jiehui Deng, Xiuhua Chen, Jianxin $\mathrm{Wu}$, and Jian Yang. Deep discriminative CNN with temporal ensembling for ambiguously-labeled image classification. In Proc. of AAAI, 2020.

[Yu and Zhang, 2016] Fei Yu and Min-Ling Zhang. Maximum margin partial label learning. In $A C M L, 2016$.

[Zeng et al., 2013] Zinan Zeng, Shijie Xiao, Kui Jia, TsungHan Chan, Shenghua Gao, Dong Xu, and Yi Ma. Learning by associating ambiguously labeled images. In Proc. of CVPR, 2013.

[Zhang et al., 2016] Min-Ling Zhang, Bin-Bin Zhou, and $\mathrm{Xu}$-Ying Liu. Partial label learning via feature-aware disambiguation. In Proc. of KDD, 2016.

[Zhang et al., 2017] Min-Ling Zhang, Fei Yu, and CaiZhi Tang. Disambiguation-free partial label learning. IEEE Transactions on Knowledge and Data Engineering, 29(10):2155-2167, 2017.

[Zhao et al., 2017] Zhengli Zhao, Dheeru Dua, and Sameer Singh. Generating natural adversarial examples. arXiv preprint arXiv:1710.11342, 2017. 